\title{
IMPACTO DEL TRABAJO JUVENIL SOBRE EL ESTADO DE SALUD DE LOS JÓVENES EN COLOMBIA*
}

Recibido: 25 de abril de 2012 • Aceptado: 15 de octubre de 2013

Willis Ruiz Marín**

\section{RESUMEN}

Este trabajo tiene por objetivo determinar el impacto del trabajo juvenil en la salud de los menores en Colombia. Para mostrar el efecto del trabajo juvenil en la salud se utiliza la información de la encuesta de calidad de vida de 2008 y se estiman modelos econométricos, logit ordenados. Se obtiene como resultado que el trabajo juvenil cuando se desarrolla sin las medidas adecuadas, se constituye en un problema que afecta el bienestar de los jóvenes colombianos.

\section{PALABRAS CLAVE}

Trabajo juvenil; percepción del estado de salud; logit ordenado

\section{CLASIFICACIÓN JEL} $\mathrm{J} 49, \mathrm{~J} 40, \mathrm{~J} 28, \mathrm{I} 15$

\section{CONTENIDO}

Introducción; 1. Estado del arte; 2. Los datos y el modelo; 3. Resultados;

4. Conclusiones y recomendaciones; Bibliografía.

Este artículo es producto del proyecto de investigación "Impacto del trabajo juvenil en la percepción del salud de los jóvenes colombianos", presentado como requisito para optar al título de Magíster en Economía de la Universidad del Norte. Grupo Desarrollo Económico, Instituciones y Políticas Públicas para el Progreso del Caribe Colombiano (PROCARIBE), Clasificado en la categoría D de Colciencias, Convocatoria 2010. La investigación fue financiada con recursos propios del grupo y su ejecución se llevó a cabo entre junio de 2011 y marzo de 2012.

* Economista, Universidad del Atlántico, Barranquilla, Colombia. Magíster en Economía, Universidad del Norte, Barranquilla, Colombia. Profesor Facultad de Ciencias Económicas, Universidad de la Salle, Bogotá, Colombia. Funcionario del Ministerio del Trabajo, Bogotá, Colombia. Correo electrónico: wruiz@unisalle. edu.co o wruiz@mintrabajo.gov.co 


\section{HEALTH EFFECTS OF YOUTH LABOR IN COLOMBIAN YOUNGSTERS ABSTRACT}

The objective of this paper is to determine health effects that youth labor has in under ages in Colombia. In order to demonstrate the health effects due to child labor, the following information was used: The 2008 quality of life survey, econometric models and ordered logit models. The results concluded that when the labor is carried out without following the necessary requirements, it constitutes a problem that affects the wellbeing of Colombian youngsters.

\section{KEY WORDS}

Youth labor; health condition perception; ordered logit model

\section{JEL CLASSIFICATION}

J49, J40, J28, I1 5

\section{CONTENT}

Introduction; 1. State of art; 2. Data and the model; 3. Results; 4. Conclusions and recommendations; Bibliography.

\section{IMPACTO DO TRABALHO JUVENIL SOBRE O ESTADO DE SAÚDE DOS JOVENS NA COLÔMBIA}

\section{RESUMO}

Este trabalho tem por objetivo determinar o impacto do trabalho juvenil na saúde dos menores na Colômbia. Para mostrar o efeito do trabalho juvenil na saúde se utiliza a informação da enquete de qualidade de vida de 2008 e se estimam modelos econométricos, logit ordenados. Obtém-se como resultado que o trabalho juvenil quando se desenvolve sem as medidas adequadas, constitui-se num problema que afeta o bem-estar dos jovens colombianos.

\section{PALAVRAS CHAVES}

Trabalho juvenil; percepção do estado de saúde; logit ordenado

\section{CLASSIFICAÇÃO JEL}

J49, J40, J28, I15.

\section{CONTEÚDO}

Introdução; 1. Estado da arte; 2. Os dados e o modelo; 3. Resultados; 4. Conclusões e recomendações; Bibliografia. 


\section{INTRODUCCIÓN}

El trabajo juvenil, cuando se lleva a cabo sin las condiciones mínimas requeridas afecta el desarrollo social de los menores y de sus familias e incide en las oportunidades para adquirir habilidades y destrezas que les permitan desenvolverse en un ambiente acorde con su etapa de desarrollo. Es por ello que el propósito de la presente investigación es el de indagar por el impacto que tiene el trabajo juvenil sobre el bienestar de los menores.

Se utiliza la información de la encuesta de calidad de vida del año 2008 (en adelante, ECV-2008), y se estiman tres modelos econométricos con el fin de determinar la relación entre las variables familiares y sociales, y el trabajo juvenil. El primer modelo se estima usando como variable de control el rango de edad de 12 a 17 años. El segundo modelo se estima usando como variable de control el género femenino, es decir, se hace un análisis para las jóvenes trabajadoras, y el tercer modelo se estima usando como variable de control el género masculino.

En el tema de protección de los menores, en el curso del siglo XX, se han adoptado varias iniciativas internacionales como la creación de la Organización Internacional del Trabajo (OIT) en 1919, la declaración de Ginebra en 1924, la creación de la UNICEF en 1946, el establecimiento de la edad mínima para laborar en 1973, la declaración de las Naciones Unidas sobre los derechos de los niños en 1989 y el convenio de la Organización Internacional del Trabajo para combatir las peores formas del trabajo infantil en 1999. Todas estas iniciativas, resaltan Kolk y Tulder (2002), han puesto de relieve la importancia de este fenómeno y no ahorran esfuerzos para combatir y minimizar el trabajo infantil y juvenil.

Sin embargo, es a partir de la ratificación de la convención de la ONU de 1989 (ONU, 1989) sobre los derechos de los niños, cuando los gobiernos y la sociedad civil comienzan a tomar mayor conciencia acerca de la importancia de la protección a la infancia y del impacto negativo del trabajo infantil y juvenil. La última década del siglo XX se caracteriza por la proliferación de investigaciones en torno a la problemática del trabajo juvenil y los efectos en la salud de los menores, así como por la adopción de políticas y programas sociales encaminados a la abolición de estas prácticas.

Un aspecto relevante relacionado con la problemática del trabajo realizado por los jóvenes es el hecho de contar con información disponible para profundizar y conocer a fondo el fenómeno. Al respecto, la UNICEF (2003, p. 33) argumenta que "no existe continuidad en la recolección y sistematización de la información sobre la situación laboral de los menores de 14 años; en el año 1999 se realizó el último esfuerzo en esa dirección, al 
incluir el Módulo Especial sobre Trabajo Infantil en la Encuesta Continua de Hogares del Instituto Nacional de Estadística de Uruguay - INE-".

Por otro lado, la OIT (2007a) establece que Colombia es uno de los países que ratificó los convenios referentes a la abolición del trabajo realizado por menores de 18 años. El Convenio 138 de 1973 (OIT, 1973), sobre la edad mínima de admisión a cualquier trabajo o empleo, fue suscrito por Colombia apenas a partir del año 2001, y el Convenio 182 de 1999 (OIT, 1999) sobre la prohibición y eliminación de las peores formas de trabajo infantil, en 2005.

El objetivo de la presente investigación es analizar las consecuencias del trabajo juvenil en la percepción del estado de salud de los menores en Colombia, motivado por la relevancia que pueda tener este tema para el bienestar de estos. El hecho de que los menores realicen actividades laborales les reduce el tiempo disponible que tienen para disfrutar de un desarrollo físico, mental y académico adecuado a su edad.

El presente estudio aporta evidencia empírica, apoyado en la econometría, sobre el impacto negativo del trabajo juvenil en la percepción del estado de salud de los menores colombianos que realizan actividades laborales. En el caso de Colombia se han adelantado varias investigaciones sobre este tema, las cuales han hecho aportes significativos para entender la naturaleza de este fenómeno. Flórez, Knaul y Méndez (1994) señalan que son escasas las investigaciones empíricas que cuantifican la magnitud de la problemática del trabajo infantil y juvenil.

El artículo ha sido dividido en cinco partes: la introducción; revisión de la literatura sobre trabajo juvenil; los datos y el modelo; resultados econométricos de los efectos del trabajo juvenil en la salud, y las conclusiones y recomendaciones del estudio.

\section{ESTADO DEL ARTE}

El trabajo de los menores es un fenómeno que afecta a la sociedad en la actualidad. La OIT (2008) considera que algunos de los menores que trabajan lo hacen en condiciones inadecuadas; es por ello, que organismos internacionales hacen constantes esfuerzos con el fin de minimizar el impacto que tiene este sobre el bienestar de los menores en su estado de salud.

Dentro de las principales organizaciones se encuentran: la Organización de las Naciones Unidas (ONU), la Organización Internacional del Trabajo (OIT) y la Organización Mundial de la Salud (OMS). En el presente estudio se utiliza la definición de 
trabajador juvenil de Pedraza y Ribero (2006), que cobija el rango de edad de 12 a 17 años, y que durante el período de referencia (en octubre se recogió la información de la ECV-2008) se encontraban en una de las siguientes situaciones: en ejercicio de una actividad en la producción de bienes y servicios, de por lo menos una hora remunerada a la semana; en trabajo familiar sin remuneración, por lo menos 15 horas a la semana, y los que estaban vinculados a un proceso de producción cualquiera sin remuneración).

Pedraza y Ribero (2006), en las investigaciones sobre trabajo de los menores de edad, manejan los siguientes enfoques: el enfoque del mercado laboral, el cual se desarrolla cuando se pensaba que el trabajo de los menores podía sustituir el trabajo de los adultos; el enfoque del capital humano que señala que el trabajo de los menores es una característica de las economías subdesarrolladas, y que debe ser erradicado para que estos alcancen una acumulación de capital humano y buenos estados de salud que les permitan ser unos adultos con proyección de vida y bienestar.

Estos autores señalan que el tercer enfoque que aborda la problemática del trabajo de los menores es el de la responsabilidad social, según la cual los menores no deben ser maltratados cuando participan en el mercado laboral. Por el contrario, lo que se debe hacer es protegerlos de los abusos sin excluirlos de las actividades laborales, es decir, esta es otra visión que indica que las actividades laborales de los menores no se ven de una forma crítica, cuando se les dan las condiciones mínimas requeridas, sumada al enfoque del pensamiento del trabajo infanto-juvenil que plantea los derechos y el bienestar de los trabajadores más jóvenes como el punto más relevante. Por lo tanto, la solución planteada para el trabajo juvenil es un conocimiento amplio de los menores, donde ellos mismos participen en las iniciativas para contrarrestar el problema del trabajo infantil y juvenil.

A mediados de la década de los noventa, en un estudio realizado por Flórez, Knaul, y Méndez (1994) en Colombia, se encuentra que el número de jóvenes que trabajan es elevado y que las condiciones laborales no son las adecuadas; las jornadas laborales son excesivas, la remuneración es baja y se enmarcan por lo general en el sector informal. Los hallazgos de los autores muestran que el trabajo juvenil aumenta el riesgo de deterioro del estado de salud de los menores.

Feldman, García y Araldsen (1997) encuentran que el trabajo juvenil constituye una amenaza para la salud del menor cuando las actividades laborales son realizadas en situaciones peligrosas, como el trabajo en las minas o el manejo de pesticidas. Lo anterior trae como consecuencia la perturbación de su crecimiento saludable. 
En otro estudio realizado por Mosquera, Romero y Huayhua, (1998) en Perú, en relación con el trabajo juvenil en la minería artesanal, se evidencia que el número de horas dedicadas por los menores a las actividades laborales en la mina supera el tiempo que estos resisten de acuerdo con sus fuerzas. Como consecuencia del gran esfuerzo físico, los menores padecen de dolores de cabeza, de extremidades y de espalda, así como de enfermedades respiratorias por la exposición al polvo y a elementos químicos como el mercurio, utilizado para separar el oro de otros minerales. Mosquera, Romero y Huayhua $(1998$, p. 6) lo describen de la siguiente forma:

Los riesgos sobre la salud se incrementan cuando los menores deben realizar trabajos, manipular sustancias o utilizar herramientas que implican riesgos de accidentes o enfermedades, y se encuentran desprovistos de protección. Esto es evidente en el caso de actividades mineras como las realizadas en Santa Filomena, particularmente en los trabajos de extracción artesanal, acarreo y tratamiento artesanal del mineral, en que los menores comparten con los adultos los riesgos inherentes a estas labores que se desarrollan sin medios de protección.

Lo anterior implica que las condiciones inadecuadas del trabajo son las que afectan a los niños, y que probablemente si los menores laboraran bajo condiciones más adecuadas de trabajo este podría verse como una opción de progreso. Sin embargo, en este estudio se considera como condición ideal que los menores se dediquen exclusivamente a estudiar, ya que de esta forma adquirirán las habilidades para desarrollarse adecuadamente en la sociedad.

Rodríguez (2000), en relación con la protección social de los menores trabajadores, señala que los jóvenes que trabajan lo hacen en sectores de alta informalidad, no gozan de protección social, los empleos son mal remunerados, precarios e inestables en comparación con los de los adultos.

En otro estudio sobre trabajo juvenil en el sector minero, realizado por la OIT (2001a) en Colombia, en el municipio de Condoto, Chocó, se encontró que los menores están expuestos a enfermedades por el lugar en que llevan a cabo sus actividades laborales: la mina de oro. La enfermedad más padecida por los menores trabajadores en esta zona es el paludismo, aunque se presentan otras enfermedades tales como: el tifo, la gripa, la diarrea, las infecciones en la piel y los parásitos.

En otra investigación realizada por la OIT (2001b) en Colombia, sobre esta misma temática, en una mina artesanal de carbón en Sogamoso, Boyacá, se encontró que las enfermedades más frecuentes que padecen los menores trabajadores son: la gripa, problemas pulmonares y de respiración y la amebiasis causada por el agua con la cual se preparan los alimentos; lo anterior, aunado a la desnutrición, debida a la carencia de una dieta alimentaria adecuada. 
Siguiendo con el trabajo juvenil en el sector minero, se encuentra un estudio hecho por la OIT (2001c) en el municipio de Muzo en Boyacá (Colombia) el cual revela que los menores dedicados a la minería artesanal de esmeraldas están expuestos a esfuerzos físicos, mala remuneración, temperaturas extremas, olores fuertes o la presencia de polvo en el ambiente, picaduras de insectos y ruidos, factores que afectan los estados de salud de los jóvenes trabajadores.

La OIT (2006), en un estudio dentro del programa internacional de eliminación del trabajo infantil en Bangladesh, Brasil y Camboya, encontró que existe una importante relación causal entre el aumento de las horas de trabajo semanal del menor y el aumento de la probabilidad de sufrir enfermedades o lesiones relacionadas con el trabajo. Se estableció también la influencia del sector en el que trabajan los menores en la naturaleza de los riesgos para la salud.

Fernández y Campos (2004), en un estudio con el apoyo de la OIT, señalan que los jóvenes trabajan el mismo número de horas que los adultos. En palabras de Fernández y Campos (2004, p. 17):

[...] la población de jóvenes que realizan actividades en el mercado laboral se ocupa principalmente en comercio, industria manufacturera, servicio doméstico y servicios personales; la duración de las jornadas de trabajo y continuidad del trabajo resultan similares a las constatadas para los trabajadores adultos, especialmente en las ramas donde los jóvenes trabajan en forma mayoritaria.

Esta situación afecta el estado de salud de los jóvenes.

Vargas (2005), con el apoyo de la OIT, en el marco del programa de erradicación del trabajo infantil, estudia el peligro que entraña el trabajo de los menores en el área urbana de Panamá. Dentro de los principales resultados de la investigación se destaca que los menores laboran bajo escasas medidas de seguridad.

Según Vargas (2005), las principales actividades realizadas por los menores son: labores en supermercados, secretarios de bus, lavadores de autos, vendedores ambulantes, pepenadores (recicladores), labores agropecuarias, en construcciones y en el sector manufacturero. Estas actividades resultan peligrosas para los menores, más aún cuando estos no se protegen. Además, 50 \% de estos menores iniciaron actividades laborales entre los 4 y los 10 años de edad. De ellos, 28 \% declaró haber sufrido alguna enfermedad o lesión a causa del trabajo, entre ellas: infecciones respiratorias, dolor de cabeza, dolor de espalda, dolor de cuello, dolor de piernas, infecciones en la piel, caídas y golpes. Este estudio muestra la complejidad del trabajo juvenil en Panamá, lo que implica un impacto negativo en los estados de salud de los jóvenes trabajadores y un futuro no muy promisorio. 
Castro (2003) estudia el fenómeno de las actividades laborales por parte de los menores colombianos en la minería artesanal. Señala la importancia de combatir la explotación laboral de los jóvenes que desarrollan actividades en las minas y la industria textil, debido a que estas actividades resultan peligrosas y dañinas para la salud.

Los anteriores trabajos coinciden en afirmar que la exposición de los jóvenes a labores peligrosas, mal remuneradas, sin protección social, largas y extenuantes jornadas de trabajo generan un impacto negativo en el estado de salud de los menores.

Abdala (2001), en una investigación realizada para Uruguay, señala que el trabajo de los menores tiene un efecto perjudicial en su nivel de bienestar y que estos se deben dedicar a las actividades académicas y de ocio, propias de su edad. Al respecto, Kassouf, Mckee y Mossialos (2001), encuentran en un estudio realizado en Brasil, que el hecho de que los jóvenes entren a participar en el mercado laboral a temprana edad hace que sea menor la probabilidad de que reporten un buen estado de salud en la edad adulta.

Según Ruiz (2001), los menores trabajan bajo dudosas condiciones de contratación, extensas jornadas laborales, carencia de seguridad social y protección. La imposibilidad de estudiar, la falta de descanso, los malos tratos y los abusos sexuales, la baja o inexistente remuneración, y las relaciones de servidumbre o esclavitud les impiden alcanzar un estado de salud óptimo cuando sean adultos.

Moreno (2003) encuentra que las consecuencias más comunes de la vinculación temprana al mercado laboral son: golpes, heridas superficiales, fracturas, quemaduras, luxaciones, esguinces y otro tipo de heridas. Las enfermedades de mayor incidencia relacionadas con el trabajo de los más jóvenes son las infecciones y alergias respiratorias. Sin embargo, señala otras enfermedades que padecen los jóvenes trabajadores, tales como intoxicaciones, convulsiones, parálisis, y temblor en manos y cuerpo.

Venegas (2004) establece que los jóvenes vinculados al mercado laboral lo hacen de manera informal bajo condiciones no siempre legales ni seguras, lo que trae consecuencias negativas para la salud y pocas oportunidades de desarrollo académico.

Briceño y Pinzón (2004) hallaron que los menores, al estar en un proceso dinámico de crecimiento y desarrollo, presentan características que los hacen más propensos que los adultos a los factores de riesgo laboral.

Al respecto Mendoza (2004), en un estudio conjunto con la OIT sobre el trabajo realizado por los menores en la central de abastos de Bogotá, Corabastos, afirma 
que la vinculación temprana de los jóvenes al mercado laboral en labores peligrosas altera su crecimiento integral y armónico. Esto se traduce en un impacto negativo en la salud y el bienestar de los jóvenes trabajadores en la actualidad, y en su edad adulta. Tal como lo expresa Mendoza (2004, p. 68):

Si se analiza lo que significa para el organismo de un niño o una niña el impacto que le provoca tener que realizar actividades físicas esforzadas en condiciones climáticas y medio ambientales adversas, según sea su edad, y admitiendo que tienen condiciones de vida y alimentación por debajo de los requerimientos básicos hay que hablar de alto riesgo de enfermar de afecciones frecuentes y graves, así como de posibles secuelas derivadas de ello; todas estas eventualidades pueden trastornar el desarrollo corporal y pondoestatural de las niñas y los niños de diversas maneras.

En el mismo sentido, Straub y Rosati (2006), en Guatemala, realizan pruebas sobre los efectos a largo plazo del trabajo de los menores. Mediante el uso de la información retrospectiva sobre la edad de entrada en el mercado de trabajo, los autores analizan los efectos del trabajo juvenil en la salud de los adultos. Sostienen los autores que los efectos estimados muestran que el trabajo juvenil aumenta la probabilidad de tener mal estado de salud en la edad adulta, en un $40 \%$. La importancia y el tamaño de este resultado parece indicar que los efectos del trabajo juvenil en la salud toman tiempo en manifestarse, y que mirar las consecuencias inmediatas y de mediano plazo puede llevar a una subestimación considerable de los costos de bienestar ocasionados a los menores que realizan actividades laborales.

Pedraza y Ribero (2006), estiman un modelo logit ordenado en el cual la variable endógena es la percepción del estado de salud, el cual puede ser: malo, regular, bueno y muy bueno. Los principales resultados de esta investigación son los siguientes: se encuentra un impacto negativo en la salud de los jóvenes colombianos que se vinculan al mercado laboral; las pruebas econométricas permiten inferir que el trabajo de los jóvenes está relacionado con estados de salud regulares y malos. Por lo tanto, el trabajo de los menores se asocia con un deterioro del bienestar.

Según la OIT (2007b), en un estudio llevado a cabo en Colombia sobre el trabajo juvenil doméstico en hogares de terceros, se encontró que los jóvenes están expuestos a condiciones que los hacen vulnerables, tales como convivir con personas extrañas, vivir en el mismo puesto de trabajo, el aislamiento, las jornadas de trabajo y las actividades que superan lo permitido para menores de edad, que afectan la salud de los jóvenes. Esto genera un desprendimiento temprano de los lazos familiares y la ruptura de los procesos de construcción, de seguridad básica y autoestima. Lo anterior, aunado al hecho de que la mayoría de estos jóvenes no se encuentra afiliado al sistema de salud por intermedio del empleador, constituye un riesgo para la salud de los jóvenes que realizan esta clase de trabajos. 
Para la OIT (2009), participar en el mercado laboral obstaculiza el desarrollo físico, mental, espiritual, moral y social de los individuos y sociedades. En América Latina existen 17 millones de menores entre 5 y 17 años de edad que no están realizando las actividades propias de su edad, por estar inmersos en actividades laborales. En países como Ecuador y Bolivia el $50 \%$ de los menores indígenas entre 5 y 17 años de edad realizan actividades laborales peligrosas. Tal como lo indica la OIT (2009, p. 9):

Si bien los niños indígenas llevan a cabo tareas en su entorno familiar y comunitario que son culturalmente aceptadas y a través de las cuales reproducen el conocimiento tradicional y aprenden a desenvolverse en su medio, una enorme proporción de ellos lleva a cabo, fuera de su entorno comunitario, actividades de trabajo por cuenta ajena, en plantaciones agrícolas, minas y canteras, en el servicio doméstico o en otras formas de explotación económica y laboral. Las causas de esta situación remiten a la pobreza, discriminación, erosión cultural y ausencia de una educación de calidad y culturalmente relevante.

Lo anterior representa un alto riesgo para la salud y el bienestar de estos menores, los cuales pueden padecer peores estados de salud cuando sean adultos.

Urueña, Cuevas y Castillo (2009) señalan que el problema relacionado con el trabajo de los menores de edad ha estado asociado a los países de economías emergentes. Estiman un modelo probit bivariado y concluyen que el hecho de que los menores se vinculen al mercado laboral tiene repercusiones negativas en el bienestar de los jóvenes. Por su parte, Hernández (2009) establece que los efectos del trabajo sobre la salud de los menores son más graves que en los adultos, pues afectan su desarrollo físico y mental, y generan graves repercusiones en el futuro.

La OIT (2010), en un estudio sobre la magnitud y características del trabajo realizado por menores, señala que los jóvenes trabajadores sufren de cansancio, agotamiento y se afectan psicológicamente al desempeñar tareas inapropiadas para su edad y desarrollo, lo cual se traduce en el deterioro de los estados de salud. Además, la OIT (2011, pp. 13-14) señala que los menores dedicados a trabajos peligrosos no tienen la madurez física ni mental para desempeñar estas actividades que implican riesgo a su integridad, específicamente situaciones como cortes con cuchillos, y ceguera, debida a trabajos de soldadura. Lo cual describen de la siguiente forma:

Es posible que el fabricante de zapatos de 16 años no sepa que el pegamento que utiliza contiene benceno y esto puede conllevar el desarrollo de leucemia y la muerte a los 30 años. Los menos conocidos son los peligros vinculados con las amenazas psicosociales. Estrés, largas jornadas, trabajo a ritmo acelerado, aislamiento, intimidación, por mencionar solo unos pocos; todos ellos pueden ocasionar heridas internas, heridas que no se curan fácilmente.

Harsch (2001) en un estudio en Burkina Faso, África, señala que es usual que los 
menores de 14 años de edad se desprendan de las familias y se encaminen a otras partes del país en busca de trabajo, unas veces voluntariamente, en otros casos por insistencia de los padres, como forma para escapar de la pobreza extrema de los lugares de orígenes. Estos jóvenes, en la mayoría de casos, consiguen trabajo penoso y mal remunerado en plantaciones o en el servicio doméstico, que ponen en riesgo su salud.

En la tabla 1 se presenta un resumen de los principales aportes científicos relacionados con el impacto del trabajo juvenil en la salud de los menores, cuando este se realiza en condiciones precarias.

Tabla 1. Estado del arte sobre trabajo juvenil

\begin{tabular}{|l|c|l|l|}
\hline \multicolumn{1}{|c|}{ Autor } & Año & \multicolumn{1}{c|}{ Lugar } & \multicolumn{1}{c|}{ Resultado s principales } \\
\hline $\begin{array}{l}\text { Flórez y } \\
\text { otros }\end{array}$ & 1994 & Colombia & $\begin{array}{l}\text { El número de jóvenes que trabajan es alto y las condicio- } \\
\text { nes laborales no son adecuadas }\end{array}$ \\
\hline OIT & 1998 & Perú & $\begin{array}{l}\text { El número de horas dedicadas por los menores a las } \\
\text { actividades labórale; en la mina superan el tiempo que } \\
\text { estos resisten de acuerdo a sus fuerzas }\end{array}$ \\
\hline Rodríguez & 2000 & $\begin{array}{l}\text { A m éric a a } \\
\text { Latina }\end{array}$ & $\begin{array}{l}\text { Los jóvenes que trabajan lo hacen en sectores de alta } \\
\text { informalidac y no cuentan con protección del sistema de } \\
\text { seguridad social }\end{array}$ \\
\hline OIT & 2001 a & Colombia & $\begin{array}{l}\text { Los jóvenes trabajadores están expuestos a enferme- } \\
\text { dacfes pa las actividades laboralesrealizadas en la mina } \\
\text { del oro. lo cual les genera enfermedacfes como paludismo, } \\
\text { tifo, gripa, diarrea, infecciones en la piel y parásitos }\end{array}$ \\
\hline Abdala & 2001 & Uruguay & $\begin{array}{l}\text { El trabaje juvenil tiens un efecto perjudicial sobre el } \\
\text { bienestai de los menores }\end{array}$ \\
\hline OIT & $2001 b$ & Colombia & $\begin{array}{l}\text { sobre los riesgos en la salud de los jóvene debido a la } \\
\text { participaciór en actividades laborales en la Minería Arte- } \\
\text { sanal de Esmeraldas }\end{array}$ \\
\hline $\begin{array}{l}\text { Kassouf y } \\
\text { otros }\end{array}$ & 2001 & Brasil & $\begin{array}{l}\text { El hechc que los jóvene; entrai a participar en el mercado } \\
\text { laboral a tempranaedad, reduce la probabilidad de que } \\
\text { reportar buen estado de salud en la edad adulta. }\end{array}$ \\
\hline Ruiz & 2001 & Colombia & $\begin{array}{l}\text { El trabaje realizado por menores de edad les impide } \\
\text { alcanzai un buen estado de salud }\end{array}$ \\
\hline Harsch & 2001 & $\begin{array}{l}\text { Burkina } \\
\text { Faso }\end{array}$ & $\begin{array}{l}\text { La mayoría de los trabajadores jóvenes realizan activi- } \\
\text { dades penosas y mal remunerada; en plantaciones o en } \\
\text { el servicio doméstico lo cual aumenta el riesgo que los } \\
\text { menores padezcan peores estados de salud }\end{array}$ \\
\hline
\end{tabular}




\begin{tabular}{|c|c|c|c|}
\hline Autor & Año & Lugar & Resultado s principales \\
\hline Moreno & 2003 & Honduras & $\begin{array}{l}\text { Debido al trabajo juvenil, los menores sufrer golpes, } \\
\text { heridas superficiales fracturas quemaduras, luxaciones } \\
\text { esguinces y otro tipo de heridas afectando esto su salud }\end{array}$ \\
\hline Venegas & 2004 & $\begin{array}{l}\text { El Salva- } \\
\text { dor }\end{array}$ & $\begin{array}{l}\text { El trabajo realizado por jóvenes aumenta el riesgo de } \\
\text { padece problemas de salud. }\end{array}$ \\
\hline $\begin{array}{l}\text { Briceño y } \\
\text { Pinzón }\end{array}$ & 2004 & Colombia & $\begin{array}{l}\text { Los jóvenes trabajadores están más propensos que los } \\
\text { adultos a los factores de riesgos laborales lo cual consti- } \\
\text { tiye una amenaza para su estado de salud. }\end{array}$ \\
\hline OIT & 2006 & $\begin{array}{l}\text { Bangla- } \\
\text { desh, } \\
\text { Brasil y } \\
\text { Camboya }\end{array}$ & $\begin{array}{l}\text { Cuando los jóvenes realizar actividades labórale; aumenta } \\
\text { la probabilidad de que sufran enfermedades }\end{array}$ \\
\hline Mendoza & 2004 & Colombia & $\begin{array}{l}\text { La vinculación temprana de los jóvenes al mercado laboral } \\
\text { en actividades peligrosas afecta negativamente el creci- } \\
\text { miento integral y armónico de los menores }\end{array}$ \\
\hline $\begin{array}{lll}\text { F } & \text { e } & \text { r } \\
\text { ná } & \text { c c fe } & \text { z } \\
\text { y } & \text { Campos }\end{array}$ & 2004 & Uruguay & $\begin{array}{l}\text { Los jóvenes laboran largas jornadas similai a la de los } \\
\text { adultos lo cual tiene un efecto negativo en el estado de } \\
\text { salud de los menores }\end{array}$ \\
\hline Vargas & 2005 & P anamá & $\begin{array}{l}\text { los menores realizan sus actividades labórale; bajo escasa; } \\
\text { medidas de seguridad y en sectores peligrosos lo cual } \\
\text { afecta su estado de salud }\end{array}$ \\
\hline $\begin{array}{l}\text { Straub y } \\
\text { Rosati }\end{array}$ & 2006 & Guatemala & $\begin{array}{l}\text { El trabaje juvenil aumenta la probabilidac de tener mal } \\
\text { estadc de salud en la edad adulta en aproximadamente } \\
\text { un } 40 \text { pa ciento }\end{array}$ \\
\hline $\begin{array}{l}\text { Pedraza y } \\
\text { Ribero }\end{array}$ & 2006 & Colombia & $\begin{array}{l}\text { Encuentran que el trabajo realizado por los jóvene co- } \\
\text { lombianos deteriora la percepción del estado de salud }\end{array}$ \\
\hline OIT & 2007 & Colombia & $\begin{array}{l}\text { El trabaje juvenil hace que los menores sean vulnerables } \\
\text { y se afecte su saluc }\end{array}$ \\
\hline OIT & 2009 & $\begin{array}{l}\text { América } \\
\text { Latina }\end{array}$ & $\begin{array}{l}\text { El trabajo juvenil obstaculiza el desarrolle fisico, mental } \\
\text { espiritual moral o social de los individuos y sociedades } \\
\text { en la Mayor parte de las regiones del munde }\end{array}$ \\
\hline $\begin{array}{l}\text { Urueña y } \\
\text { otros }\end{array}$ & 2009 & Colombia & $\begin{array}{l}\text { Si los menores se vincular prematuramaite al mercado } \\
\text { labora tiene repercusiones negativas en su bienestar }\end{array}$ \\
\hline OIT & 2011 & Uruguay & $\begin{array}{l}\text { El trabajo juvenil afecta negativamente la salud de los } \\
\text { menores }\end{array}$ \\
\hline
\end{tabular}

Fuente: elaboración propia 


\section{LOS DATOS Y EL MODELO}

De acuerdo con la ENCV-2008, en Colombia existe un total de 1.093.864 jóvenes con edades entre 12 y 17 años, que realizan alguna actividad remunerada o no, en el proceso de producción de bienes y servicios. El 55,80 \% de estos jóvenes son de género masculino y el 44,20\% restante de género femenino.

La muestral corresponde a 13860 hogares, y un total de 50542 personas. La unidad de observación está conformada por los hogares colombianos; la unidad de muestreo es el segmento o conglomerado, conformado en promedio por diez hogares, y la unidad de análisis está constituida por las viviendas, los hogares y las personas. Esta encuesta contiene información relativa a la participación del trabajo juvenil, los niveles educativos de los miembros de las familias y otras características específicas de los hogares. Para el caso concreto del presente trabajo, se utilizará la información de nueve regiones: Bogotá, Antioquia, Valle del Cauca, Atlántica, Oriental, Central, Pacífica, Orinoquía y San Andrés (ver tabla 2).

En Colombia en 2001, el DANE realizó la Encuesta Nacional de Trabajo Infantil, y en 2003 incluyó el módulo especial de trabajo infantil en la encuesta de calidad de vida, ECV-2003. El DANE hace el seguimiento a los indicadores del trabajo infantil, a través de la inclusión de los módulos especiales sobre trabajo de los menores en el último trimestre de 2003 y 2005, de la Encuesta Continua de Hogares, y el módulo de trabajo infantil en la Gran Encuesta Integrada de Hogares en el cuarto trimestre de 2007, aunque en la ECV-2008 no se incluye este módulo.

Lo anterior constituye un retroceso en términos de disponibilidad de información en un tema tan relevante como el del trabajo realizado por los menores de edad, debido a que afecta el bienestar de los jóvenes. La situación del trabajo juvenil se presenta en las siguientes tablas, así: en la tabla 3 se muestran las variables y definiciones utilizadas en el presente estudio, y en la tabla 4 se muestran las estadísticas descriptivas.

Tabla 2. Jóvenes trabajadores según región

\begin{tabular}{|l|c|c|c|c|c|}
\hline \multirow{2}{*}{ Variables } & \multicolumn{5}{|c|}{ Jóvenes trabajadores por región } \\
\cline { 2 - 7 } & Masculino & Femenino & Total & \% Masculino & \% Femenino \\
\hline Atlántica & 523 & 533 & 1.056 & 7,89 & 8,04 \\
\hline Oriental & 435 & 431 & 866 & 6,56 & 6,50 \\
\hline
\end{tabular}

El autor se compromete con los lectores que así lo deseen a entregarles la base de datos en formato Stata Data (*.dta). 
Willis Ruiz Marín

\begin{tabular}{|l|c|c|c|c|c|}
\hline \multirow{2}{*}{ Variables } & \multicolumn{5}{|c|}{ Jóvenes trabajadores por región } \\
\cline { 2 - 6 } & Masculino & Femenino & Total & \% Masculino & \% Femenino \\
\hline Central & 500 & 493 & 993 & 7,54 & 7,43 \\
\hline Pacífica & 574 & 563 & 1.137 & 8,66 & 8,49 \\
\hline Bogotá & 225 & 210 & 435 & 3,39 & 3,17 \\
\hline San Andrés & 87 & 60 & 147 & 1,31 & 0,91 \\
\hline Orinoquía & 113 & 137 & 250 & 1,70 & 2,07 \\
\hline Antioquia & 487 & 464 & 951 & 7,34 & 6,99 \\
\hline Valle de Cauca & 401 & 396 & 797 & 6,05 & 5,97 \\
\hline TOTALES & 3.345 & 3.287 & 6.632 & 50,44 & 49,56 \\
\hline
\end{tabular}

Fuente: elaboración propia con datos de la ECV-2008, DANE

Tabla 3. Variables empleadas en el trabajo

\begin{tabular}{|c|c|}
\hline Variable & Definición \\
\hline Estado de salud joven & $\begin{array}{l}\text { Variable ordenada que toma el valor de 1, 2, } 3 \text { o } 4 \text { si } \\
\text { el menor reporta un estado de salud, malo, regular, } \\
\text { bueno o muy bueno respectivamente. }\end{array}$ \\
\hline Trabajo juvenil & $\begin{array}{l}\text { Variable dummy que toma el valor de } 1 \text { si el joven } \\
\text { trabaja y } 0 \text { en otro caso. }\end{array}$ \\
\hline Enfermedad en los últimos 30 días & $\begin{array}{l}\text { Variable dummy que toma el valor de } 1 \text { si el joven } \\
\text { ha presentado enfermedades en los } 30 \text { días previos } \\
\text { a la encuesta, y } 0 \text { en caso contrario. }\end{array}$ \\
\hline Edad & Años de edad del joven. \\
\hline Género masculino del joven & $\begin{array}{l}\text { Variable dummy que toma el valor de } 1 \text { si el joven } \\
\text { es de género masculino y } 0 \text { en otro caso. }\end{array}$ \\
\hline Educación universitaria padre & $\begin{array}{l}\text { Variable dummy que toma el valor de } 1 \text { si el padre del } \\
\text { joven tiene estudios universitarios y } 0 \text { en otro caso. }\end{array}$ \\
\hline Educación universitaria madre & $\begin{array}{l}\text { Variable dummy que toma el valor de } 1 \text { si la madre } \\
\text { del joven tiene estudios universitarios y } 0 \text { en otro } \\
\text { caso. }\end{array}$ \\
\hline Más de un hogar en vivienda & $\begin{array}{l}\text { Variable dummy que toma el valor de } 1 \text { si hay más } \\
\text { de un hogar en la vivienda y } 0 \text { en otro caso. }\end{array}$ \\
\hline Hogar es pobre & $\begin{array}{l}\text { Variable dummy que toma el valor de } 1 \text { si el hogar } \\
\text { se considera pobre y } 0 \text { en otro caso. }\end{array}$ \\
\hline
\end{tabular}


Impacto del trabajo juvenil sobre el estado de salud de los jóvenes en Colombia

\begin{tabular}{|l|l|}
\hline \multicolumn{1}{|c|}{ Variable } & \multicolumn{1}{|c|}{ Definición } \\
\hline Ingreso hogar & $\begin{array}{l}\text { Variable dummy que toma el valor de 1 si los in- } \\
\text { gresos del hogar no cubren los gastos mínimos y } \\
0 \text { en otro caso. }\end{array}$ \\
\hline Padece hambre el joven & $\begin{array}{l}\text { Variable dummy que toma el valor de } 1 \text { si el joven } \\
\text { padece hambre y 0 en otro caso. }\end{array}$ \\
\hline
\end{tabular}

Fuente: elaboración propia con datos de la ECV-2008, DANE

Tabla 4. Estadísticas descriptivas

\begin{tabular}{|l|c|c|c|c|}
\hline \multirow{2}{*}{\multicolumn{1}{c}{ Variables }} & \multicolumn{3}{c|}{ Jóvenes de 12 a 17 años de edad } \\
\cline { 2 - 5 } & Media & $\begin{array}{c}\text { Desviación } \\
\text { estándar }\end{array}$ & Mínimo & Máximo \\
\hline Estado de salud joven & 2,0301 & 0,5307 & 1 & 4 \\
\hline Trabajo juvenil & 0,9111 & 0,2845 & 0 & 1 \\
\hline Enfermedad en los últimos 30 días & 0,1016 & 0,3021 & 0 & 1 \\
\hline Edad joven & 14,401 & 1,6941 & 12 & 17 \\
\hline Género masculino del joven & 0,5043 & 0,5000 & 0 & 1 \\
\hline Género femenino de las jóvenes & 0,4956 & 0,5000 & 0 & 1 \\
\hline Educación universitaria madre & 3,2308 & 2,5025 & 1 & 7 \\
\hline Más de un hogar en vivienda & 0,0449 & 0,2071 & 1 & 0 \\
\hline Educación universitaria padre & 3,2191 & 2,3909 & 1 & 7 \\
\hline
\end{tabular}

Fuente: elaboración propia con datos de la ECV-2008, DANE.

La tabla 4 contiene las variables endógenas y exógenas incluidas en el estudio; el estado de salud tiene una media de 2 y una desviación típica de 0,5, y toma 4 valores de 1 a 4 . La variable trabajo juvenil toma dos valores: 1 , si el menor realiza actividades laborales, y 0 , en caso contrario. La edad media de menores es de 14 años y una desviación de 1,6.

Como se aprecia en la tabla 5 , el porcentaje de los jóvenes y las jóvenes entre 12 y 17 años de edad que trabajan es del 12,53 \% y 10,67 \%, respectivamente. Sin embargo, en cuanto a la asistencia escolar las jóvenes tienen un porcentaje de asistencia del 83,63\%, mientras que para los jóvenes es del 78,89\%. En lo referente a los estados de salud de los menores se puede destacar que el 12,53\% de los jóvenes y el 14,69 \% de las jóvenes manifestaron tener un estado de salud regular. Asimismo, el 74,02 \% de los jóvenes declaró gozar de buen estado de salud y las jóvenes revelaron gozar de buen estado de salud en un 73,53 \%. 
En el modelo econométrico Logit Ordenado, la variable endógena toma el valor de 1 , si la percepción del estado de salud es mala; 2 , si es regular; 3 , si es buena, y 4 , si es muy buena.

Tabla 5. Tasa de jóvenes trabajadores colombianos

\begin{tabular}{|l|l|c|c|c|}
\hline \multirow{2}{*}{ Categoría } & \multicolumn{3}{|c}{ Descripción } & \multicolumn{3}{c}{ Hombre } & Mujer & Total \\
\cline { 4 - 5 } Tasa de jóvenes & Jóvenes / Población total jóvenes & $50.44 \%$ & $49.56 \%$ & $100 \%$ \\
\hline $\begin{array}{l}\text { Tasa de jóvenes que } \\
\text { trabajan }\end{array}$ & $\begin{array}{l}\text { Jóvenes que trabajan / Población } \\
\text { total de jóvenes }\end{array}$ & $12.53 \%$ & $10.67 \%$ & $\begin{array}{c}\mathbf{2 3 , 2 0} \\
\%\end{array}$ \\
\hline $\begin{array}{l}\text { Tasa de jóvenes que } \\
\text { estudian }\end{array}$ & $\begin{array}{l}\text { Jóvenes que estudian / Población } \\
\text { total de jóvenes }\end{array}$ & $78.89 \%$ & $83.63 \%$ & $81.24 \%$ \\
\hline $\begin{array}{l}\text { Tasa de jóvenes que } \\
\text { trabajan y estudian }\end{array}$ & $\begin{array}{l}\text { Jóvenes que trabajan y estudian / } \\
\text { Población total de jóvenes }\end{array}$ & $4.90 \%$ & $3.79 \%$ & $8.70 \%$ \\
\hline $\begin{array}{l}\text { Tasa de jóvenes que } \\
\text { no trabajan ni estu- } \\
\text { dian }\end{array}$ & $\begin{array}{l}\text { Jóvenes que no trabajan ni estudian } \\
\text { / Población total de jóvenes }\end{array}$ & $1.02 \%$ & $0.60 \%$ & $1.62 \%$ \\
\hline $\begin{array}{l}\text { Tasa de jóvenes con } \\
\text { estado de salud muy } \\
\text { bueno }\end{array}$ & $\begin{array}{l}\text { Jóvenes con estado de salud muy } \\
\text { bueno / Población total de jóvenes }\end{array}$ & $12.8 \%$ & $11.07 \%$ & $11.94 \%$ \\
\hline $\begin{array}{l}\text { Tasa de jóvenes con } \\
\text { estado de salud bue- } \\
\text { no }\end{array}$ & $\begin{array}{l}\text { Jóvenes con estado de salud bueno } \\
\text { / Población total de jóvenes }\end{array}$ & $74.02 \%$ & $73.53 \%$ & $73.78 \%$ \\
\hline $\begin{array}{l}\text { Tasa de jóvenes con } \\
\text { estado de salud re } \\
\text { gular }\end{array}$ & $\begin{array}{l}\text { Jóvenes con estado de salud re- } \\
\text { gular / Población total de jóvenes }\end{array}$ & $12.53 \%$ & $14.69 \%$ & $13.60 \%$ \\
\hline $\begin{array}{l}\text { Tasa de jóvenes con } \\
\text { estado de salud malo }\end{array}$ & $\begin{array}{l}\text { Jóvenes con estado de salud malo } \\
\text { / Población total de jóvenes }\end{array}$ & $0.66 \%$ & $0.70 \%$ & $0.68 \%$ \\
\hline
\end{tabular}

Fuente: elaboración propia con datos de la ECV-2008, DANE

A continuación se especifica el modelo logit ordenado:

$$
\text { Prob [Salud }=\mathrm{J}]=\Phi(\beta ; \lambda, \text { Trabajo Infantil, VI, VF, VV) }
$$

Donde:

$\mathrm{J}=1$ si la salud reportada es mala,

$\mathrm{J}=2$ si la salud reportada es regular,

J $=3$ si la salud reportada es buena,

$\mathrm{J}=4$ si la salud reportada es muy buena, 
$\Phi$ corresponde a una función de distribución normal estándar,

$\beta$ es el vector de parámetros que refleja el impacto de las variables independientes en la dependiente,

$\lambda$ es un término constante,

VI son las variables individuales de control,

VF son las variables familiares de control, y

VV son las variables de control por lugar de vivienda.

En las estimaciones realizadas se utilizó información individual de edad y género de los jóvenes, los niveles educativos del padre y de la madre, el género y variables dummies relacionadas con la composición del hogar por grupos de edad, y el tamaño de la familia.

\section{RESULTADOS}

Tabla 6. Modelo logit ordenado.

Estados de salud de los y las jóvenes trabajadores colombianos

\begin{tabular}{|l|c|c|c|c|c|}
\hline \multirow{2}{*}{ Variables } & \multirow{2}{*}{ Coeficiente } & \multicolumn{3}{|c|}{ Df / dx variable endógena estado de salud } \\
\cline { 3 - 6 } & & Salud mala & Salud regular & Salud buena & $\begin{array}{c}\text { Muy buena } \\
\text { salud }\end{array}$ \\
\hline \multirow{2}{*}{ Trabajo juvenil } & -.2027 & .0015 & .0251 & -.0080 & -.0186 \\
& $(.0983)^{* *}$ & $(.0008)^{*}$ & $(.0126)^{* *}$ & $(.0048)^{*}$ & $(.0087)^{* *}$ \\
\hline \multirow{2}{*}{ Edad } & .0228 & -.0001 & -.0027 & .0007 & .0021 \\
& $(.0252)$ & $(.0001)$ & $(.0030)$ & $(.0008)$ & $(.0024)$ \\
\hline Más de un & -.2413 & .0019 & .0311 & -.0119 & -.0211 \\
hogar viv. & $(.1841)$ & $(.0017)$ & $(.0254)$ & $(.0124)$ & $(.0148)$ \\
\hline \multirow{2}{*}{ Hambre jov. } & -.2633 & .0020 & .0330 & -.0112 & -.0239 \\
\hline \multirow{2}{*}{ Univer padre } & $(.1029)^{* *}$ & $(.0009)^{* *}$ & $(.0135)^{* *}$ & $(.0057)^{* *}$ & $(.0089)^{* * *}$ \\
\hline \multirow{2}{*}{ Rural padres } & .5195 & -.0030 & -.0528 & -.0038 & .0597 \\
\hline \multirow{2}{*}{ Pobre } & $(.2004)^{* * *}$ & $(.0011)^{* * *}$ & $(.0170)^{* * *}$ & $(.0098)$ & $(.0271)^{* *}$ \\
\hline Joven & -.2901 & .0022 & .0356 & -.0108 & -.0270 \\
masculino & $(.0889)^{* * *}$ & $(.0008)^{* * *}$ & $(.0111)^{* * *}$ & $(.0042)^{* *}$ & $(.0081)^{* * *}$ \\
\hline dingresol & $(.2603$ & .0018 & .0304 & -.0067 & -.0256 \\
& $(.0840)^{* * *}$ & $(.0007)^{* *}$ & $(.0107)^{* * *}$ & $(.0025)^{* * *}$ & $(.0095)^{* * *}$ \\
\hline
\end{tabular}


Willis Ruiz Marín

\begin{tabular}{|c|c|c|c|c|c|}
\hline \multirow[b]{2}{*}{ Variables } & \multirow[b]{2}{*}{ Coeficiente } & \multicolumn{4}{|c|}{ Df / dx variable endógena estado de salud } \\
\hline & & Salud mala & Salud regular & Salud buena & $\begin{array}{l}\text { Muy buena } \\
\text { salud }\end{array}$ \\
\hline \multicolumn{6}{|l|}{$\begin{array}{l}\text { Ancillary } \\
\text { parameters }\end{array}$} \\
\hline _ cutl & \multicolumn{5}{|c|}{-4.968946} \\
\hline _ cut2 & \multicolumn{5}{|c|}{-1.816581} \\
\hline _ cut3 & \multicolumn{5}{|c|}{2.040612} \\
\hline $\begin{array}{l}\text { Número de } \\
\text { observaciones }\end{array}$ & \multicolumn{5}{|c|}{2980} \\
\hline Pseudo R2 & \multicolumn{5}{|c|}{0.0195} \\
\hline Log Likelihood & \multicolumn{5}{|c|}{-2332.3994} \\
\hline
\end{tabular}

Notación: ${ }^{* * *}$ Significancia al 1 \%, "* Significancia al 5 7\%, " Significancia al 10 \% Errores estándar en paréntesis

Fuente: elaboración propia con datos de la ECV-2008, DANE

En la tabla 6 se observa que cuando los jóvenes trabajan existe una mayor probabilidad de reportar niveles más bajos de los estados de salud. Sin embargo, hay que tener en cuenta que la variable endógena utilizada es la percepción particular de los estados de salud de los jóvenes. La estimación arrojó relaciones importantes entre la variable endógena y las exógenas. El nivel de ingresos está relacionado con reportar estados de salud más saludables. Por el contrario, que el padre y la madre residan en áreas rurales aumenta la probabilidad de reportar niveles más bajos de salud. Si el joven trabajador es de género masculino, este tendrá más probabilidad de gozar de mejores estados de salud.

El hecho de que los jóvenes tengan mayor edad no incide en la probabilidad de tener mejores o peores estados de salud. Mientras que si el hogar se considera pobre afecta la probabilidad de reportar buenos estados de salud. En el caso de que el padre tenga estudios universitarios se aumenta la probabilidad de reportar mejores estados de salud.

Contrario a lo que se esperaba, el hecho de que la madre tenga alto nivel de formación académica no está relacionado con el hecho que los estados de salud percibidos de los menores sean mejores. Si los jóvenes padecen hambre por problemas económicos en los hogares esto se asocia con la probabilidad de tener mal estado de salud. Otro factor como la existencia de más de un hogar en la vivienda no afecta significativamente la probabilidad de tener mejor o peor estado de salud. 
Por otro lado, un factor que influye en la probabilidad de que los jóvenes padezcan peores estados de salud está relacionado con la zona donde viven los padres: si estos residen en zonas rurales se reduce la probabilidad de tener buenos estados de salud. Sin embargo, la procedencia de los mismos jóvenes no influye en la probabilidad de que los jóvenes tengan mejores o peores estados de salud.

La tabla 7 muestra los resultados del género femenino que realizan actividades laborales; se constata que el impacto del trabajo juvenil en la percepción de los estados de salud es negativo cuando se controla por edad y género femenino, que cuando se controla la estimación solo por edad. Entre los principales resultados se encuentra que cuando las jóvenes realizan trabajos se reduce en 2,1 \% la probabilidad de tener un estado de salud muy bueno. La edad no influye en la probabilidad de tener mejores o peores estados de salud en las trabajadoras jóvenes. Por otro lado, que el padre y la madre residan en áreas rurales aumenta en 1,65\% la probabilidad de reportar niveles más bajos de salud.

Si las jóvenes trabajadoras tienen mayor edad no se afecta la probabilidad de tener mejor o peor estado de salud; pero si el hogar declara ser pobre la probabilidad de reportar buenos estados de salud varía en 1,44 \% y 3,60 \%, respectivamente. En el caso de que el padre de las menores que realizan actividades laborales posea estudios superiores aumenta la probabilidad de reportar muy buen estado de salud en 7,62 \%. Otro factor como la existencia de más de un hogar en la vivienda no afecta la probabilidad de tener mejor o peor estado de salud.

Un factor que influye en la probabilidad de que las jóvenes padezcan peores estados de salud está relacionado con la zona donde viven los padres: si estos residen en zonas rurales se reduce la probabilidad de tener muy buen estado de salud en $1,24 \%$.

Tabla 7. Modelo logit ordenado.

Estados de salud de los jóvenes trabajadores de género femenino

\begin{tabular}{|l|c|c|c|c|c|}
\hline \multirow{2}{*}{ Variables } & \multirow{2}{*}{ Coeficiente } & \multicolumn{4}{|c|}{$d f / d x$} \\
\cline { 3 - 6 } & & Salud Mala & $\begin{array}{c}\text { Salud } \\
\text { Regular }\end{array}$ & Salud Buena & $\begin{array}{c}\text { Muy Buena } \\
\text { Salud }\end{array}$ \\
\hline \multirow{2}{*}{ Trabajo Juvenil } & -.2407 & .0018 & .0326 & -.0133 & -.0210 \\
& $(.1347)^{*}$ & $(.0011)$ & $(.0189)^{*}$ & $(.0089)$ & $(.0113)^{*}$ \\
\hline \multirow{2}{*}{ Edad } & .0022 & -.0000 & -.0002 & .0001 & .0002 \\
& $(.0350)$ & $(.0002)$ & $(.0045)$ & $(.0016)$ & $(.0032)$ \\
\hline
\end{tabular}


Willis Ruiz Marín

\begin{tabular}{|c|c|c|c|c|c|}
\hline \multirow[b]{2}{*}{ Variables } & \multirow[b]{2}{*}{ Coeficiente } & \multicolumn{4}{|c|}{$d f / d x$} \\
\hline & & Salud Mala & $\begin{array}{l}\text { Salud } \\
\text { Regular }\end{array}$ & Salud Buena & $\begin{array}{l}\text { Muy Buena } \\
\text { Salud }\end{array}$ \\
\hline \multirow{2}{*}{ Masdeunhogarviv } & -.1702 & .0013 & .0233 & -.0099 & -.0146 \\
\hline & $(.2421)$ & $(.0020)$ & $(.0347)$ & $(.0172)$ & (.0196) \\
\hline \multirow{2}{*}{ hambrejov } & -.2523 & .0019 & .0344 & -.0143 & -.0219 \\
\hline & $(.1450)^{*}$ & $(.0013)$ & $(.0206)^{*}$ & $(.0101)$ & $(.0120)^{*}$ \\
\hline \multirow{2}{*}{ univerpadre } & .6572 & -.0035 & -.0699 & -.0027 & .0762 \\
\hline & $(.2870)^{* *}$ & $(.0015)^{* *}$ & $(.0243)^{* * *}$ & $(.0166)$ & $(.0408)^{*}$ \\
\hline \multirow{2}{*}{ ruralpadres } & -.2718 & .0020 & .0362 & -.0139 & -.0243 \\
\hline & $(.1215)^{* *}$ & $(.0010)^{*}$ & $(.0165)^{* *}$ & $(.0072)^{*}$ & $(.0106)^{* *}$ \\
\hline \multirow{2}{*}{ pobre } & -.3784 & .0025 & .0479 & -.0144 & -.0360 \\
\hline & $(.1294)^{* * *}$ & $(.0011)^{* *}$ & $(.0159)^{* * *}$ & $(.0052)^{* * *}$ & $(.0128)^{* * *}$ \\
\hline \multirow{2}{*}{ dingresol } & -.2131 & .0015 & .0277 & -.0096 & -.0196 \\
\hline & $(.1303)^{*}$ & $(.0010)$ & $(.0169)^{*}$ & $(.0061)$ & $(.0120)$ \\
\hline \multicolumn{6}{|l|}{$\begin{array}{l}\text { Ancillary parame- } \\
\text { ters }\end{array}$} \\
\hline _ cutl & \multicolumn{5}{|c|}{-5.4544} \\
\hline _ cut2 & \multicolumn{5}{|c|}{-2.1470} \\
\hline _ cut3 & \multicolumn{5}{|c|}{1.6504} \\
\hline $\begin{array}{l}\text { Número de } \\
\text { observaciones }\end{array}$ & \multicolumn{5}{|c|}{1539} \\
\hline Pseudo R2 & \multicolumn{5}{|c|}{0.0225} \\
\hline Log Likelihood & \multicolumn{5}{|c|}{-1225.4384} \\
\hline
\end{tabular}

Notación: ${ }^{* * *}$ Significancia al $1 \%,{ }^{* *}$ Significancia al $5 \%,{ }^{*}$ Significancia al $10 \%$.

Errores estándar en paréntesis

Fuente: elaboración propia con datos de la ECV-2008, DANE

La tabla 8 muestra los resultados de los jóvenes de género masculino que desempeñan actividades laborales. Los resultados indican que cuando los jóvenes trabajan, la probabilidad de tener mejores o peores estados de salud no es estadísticamente significativa. La edad no influye en la probabilidad de tener mejores o peores estados de salud en los trabajadores jóvenes. También se encuentra que si el padre y la madre residen en áreas rurales se reduce la probabilidad de reportar buen y muy buen estado de salud en 0,67 \% y 3,01\%. 
El hecho de que los jóvenes trabajadores tengan mayor edad no incide en la probabilidad de tener mejores o peores estados de salud, mientras que si el hogar se considera pobre reduce la probabilidad de reportar percepciones de buenos estados de salud en 0,14 \% y 1,19\%, respectivamente. En el caso de que el padre de los menores que realizan actividades laborales tenga estudios universitarios no afecta la probabilidad de reportar mejor o peor estado de salud. Si existe más de un hogar en la vivienda, no se afecta significativamente la probabilidad de tener mejores o peores estados de salud.

Un factor que influye negativamente en la probabilidad de que los jóvenes padezcan peores estados de salud está relacionado con la zona donde residen los padres, si estos viven en zonas rurales disminuye la probabilidad de tener muy buen estado de salud en 3,01 \%.

Tabla 8. Modelo logit ordenado.

Estados de salud de los jóvenes trabajadores de género masculino

\begin{tabular}{|l|c|c|c|c|c|}
\hline \multirow{2}{*}{ Variables } & \multirow{2}{*}{ Coeficiente } & \multicolumn{4}{|c|}{$d f / d x$} \\
\cline { 3 - 6 } & & Salud Mala & Salud Regular & Salud Buena & $\begin{array}{c}\text { Muy Buena } \\
\text { Salud }\end{array}$ \\
\hline \multirow{2}{*}{ Trabajo Juvenil } & -.1594 & .0012 & .0177 & -.0034 & -.0155 \\
& $(.1445)$ & $(.0012)$ & $(.0164)$ & $(.0043)$ & $(.0136)$ \\
\hline \multirow{2}{*}{ Edad } & .0473 & -.0003 & -.0051 & .0007 & .0047 \\
& $(.0365)$ & $(.0003)$ & $(.0039)$ & $(.0007)$ & $(.0036)$ \\
\hline \multirow{2}{*}{ Masdeunhogarviv } & -.3313 & .0029 & .0397 & -.0132 & -.0295 \\
& $(.2832)$ & $(.0030)$ & $(.0374)$ & $(.0183)$ & $(.0223)$ \\
\hline \multirow{2}{*}{ Hambrejov } & -.2846 & .0023 & .0322 & -.0076 & -.0270 \\
& $(.1472)^{* *}$ & $(.0014)^{*}$ & $(.0175)^{*}$ & $(.0061)$ & $(.0133)^{* *}$ \\
\hline \multirow{2}{*}{ Univerpadres } & .3710 & -.0024 & -.0354 & -.0043 & .0422 \\
& $(.2804)$ & $(.0017)$ & $(.0235)$ & $(.0114)$ & $(.0359)$ \\
\hline \multirow{2}{*}{ Ruralpadre } & -.3090 & .0025 & .0343 & -.0067 & -.0301 \\
& $(.1309)^{* *}$ & $(.0013)^{* *}$ & $(.0149)^{* *}$ & $(.0047)$ & $(.0124)^{* *}$ \\
\hline \multirow{2}{*}{ Pobre } & -.1172 & .0008 & .0125 & -.0014 & -.0119 \\
& $(.1375)$ & $(.0010)$ & $(.0145)$ & $(.0017)$ & $(.0141)$ \\
\hline \multirow{2}{*}{ dingresol } & -.2562 & .0019 & .0274 & -.0034 & -.0259 \\
& $(.1353)^{*}$ & $(.0011)^{*}$ & $(.0143)^{*}$ & $(.0029)$ & $(.0138)^{*}$ \\
\hline
\end{tabular}




\begin{tabular}{|c|c|c|c|c|c|}
\hline \multirow[b]{2}{*}{ Variables } & \multirow[b]{2}{*}{ Coeficiente } & \multicolumn{4}{|c|}{$d f / d x$} \\
\hline & & Salud Mala & Salud Regular & Salud Buena & $\begin{array}{l}\text { Muy Buena } \\
\text { Salud }\end{array}$ \\
\hline \multicolumn{6}{|l|}{ Ancillary parameters } \\
\hline _ cutl & \multicolumn{5}{|c|}{-4.6013} \\
\hline _ cut2 & \multicolumn{5}{|c|}{-1.6292} \\
\hline _ cut3 & \multicolumn{5}{|c|}{2.3018} \\
\hline $\begin{array}{l}\text { Número de observa- } \\
\text { ciones }\end{array}$ & \multicolumn{5}{|c|}{1441} \\
\hline Pseudo R2 & \multicolumn{5}{|c|}{0.0157} \\
\hline Log Likelihood & \multicolumn{5}{|c|}{-1103.9252} \\
\hline
\end{tabular}

Notación: *** Significancia al 1 \%, "* Significancia al 5 \%, " Significancia al 10 \%.

Errores estándar en paréntesis

Fuente: elaboración propia con datos de la ECV-2008, DANE

\section{CONCLUSIONES Y RECOMENDACIONES}

La evidencia encontrada en el presente trabajo demuestra que el trabajo juvenil, cuando se desarrolla en condiciones poco adecuadas para los menores, se constituye en un problema que afecta el bienestar de los jóvenes colombianos. En lo relacionado con los estados de salud de los menores, se halla que el 23,21 \% trabajan, cuando estos deberían estar solo dedicados a realizar actividades propias de su etapa de desarrollo. La investigación revela un impacto negativo en la percepción de los estados de salud de los jóvenes que laboran en condiciones precarias.

Por otro lado, se encuentra que factores como el nivel de ingresos, que el trabajador juvenil sea de género masculino, que el padre tenga estudios universitarios generan una mayor probabilidad de reportar buenos estados de salud. La edad de los jóvenes y la existencia de más de un hogar en la vivienda no inciden en la probabilidad de tener mejores o peores estados de salud. Se encuentra que si el padre y la madre residen en áreas rurales, si el hogar se considera pobre y si los jóvenes padecen hambre por problemas económicos de los hogares aumenta la probabilidad de que los menores reporten niveles más bajos de los estados de salud.

Los resultados encontrados en este trabajo aportan más evidencia empírica relacionando variables de tipo familiar y social, evidencia que apoya los resultados de otros estudios nacionales e internacionales, respecto al efecto negativo del trabajo juvenil en la salud y bienestar de los menores, cuando se realiza sin las condiciones mínimas, pero explicados desde variables familiares y sociales como 
el nivel educativo de los padres, si proviene del campo o de la ciudad, el nivel de ingreso del hogar, el género del joven y la edad. Estas variables se justifican en el estudio debido a que el autor considera importante la relación entre estas y el hecho de realizar actividades laborales sin las condiciones mínimas requeridas para los menores. Estos hallazgos se pueden utilizar para implementar medidas de política económica como la inclusión de un rubro exclusivo en los presupuestos públicos del orden nacional, departamental y municipal, para apoyar a los jóvenes de escasos recursos, con el fin de que asistan a la escuela y no se dediquen a realizar actividades laborales en su infancia. De llegar a implementarse esta política, probablemente se minimizaría el efecto negativo de este fenómeno.

Los resultados de este trabajo son consecuentes con los de otros estudios, aunque hay que tener en cuenta que en el presente estudio se utilizan variables distintas a las de Pedraza y Ribero (2006), OIT (2011), OIT (2007), Castro (2003), OIT (2001) y Kassouf, Mckee y Mossialos (2001) en el hecho de que si los jóvenes realizan actividades laborales en condiciones inadecuadas aumenta la probabilidad de padecer estados de salud malos y regulares en comparación con los jóvenes que no realizan dichas actividades.

En Colombia en los años 2001, 2003, 2005 y 2007 se recopiló información relevante relacionada con las actividades laborales realizadas por los jóvenes. Sin embargo, la situación presentada en el año 2008 no debe repetirse, ya que el DANE no recopiló información relativa al trabajo infantil (5 a 7 años de edad), y solo recopiló trabajo juvenil (12 a 17 años de edad) en la Encuesta Nacional de Calidad de Vida del año 2008. Por lo tanto, se pierde información que permitiría entender este fenómeno de una manera más clara.

Las recomendaciones que se hacen en este trabajo son varias. La primera está relacionada con la creación de una política pública de apoyo a los menores de escasos recursos tanto de la ciudad como del campo, que les permita mantenerse dentro del sistema educativo y lograr progresar a través de la educación.

Otra recomendación es la creación de incentivos tributarios a las firmas del sector privado que apoyen con donaciones, recursos, construcción y mantenimiento de proyectos educativos, que beneficien a los menores.

Exigir desde el Gobierno nacional central la creación, en los niveles departamental y municipal de un rubro presupuestal exclusivo para el apoyo educativo de los menores colombianos.

Finalmente, es importante que cada país se comprometa a trabajar en la erradicación del trabajo realizado por los menores, debido al efecto que tiene este fenómeno 
en el bienestar de los jóvenes. Organizaciones como la OIT y la UNICEF trabajan en muchas regiones del mundo para proteger a los menores que se encuentran afectados por esta situación. El autor considera que en Colombia se debe recopilar, de una manera sistemática, información relativa a este fenómeno, debido a que en otros países se realizan estudios más específicos, por ejemplo, los cuantitativos que permiten conocer a fondo qué tipo de actividades laborales realizan los menores y su impacto en la salud, esto permitirá implementar medidas de política pública acordes con las dimensiones del problema.

\section{BIBLIOGRAFÍA}

Abdala, E. (2001). Un análisis acerca de los jóvenes que no trabajan ni estudian. Administración Nacional de Educación Pública, Montevideo, 70 pp.

Briceño, L. y Pinzón, Á. (2004). Efectos del trabajo infantil en la salud del menor trabajador. En: Revista Salud Pública, Vol. 6 N. 3 pp. 270-288.

Castro, A. (2003). Construcción de un modelo de monitoreo sobre trabajo infantil en la minería artesanal colombiana. Organización Internacional del Trabajo. Programa Internacional Para la Erradicación del Trabajo Infantil, Bogotá, 42 pp.

Feldman, S.; García, E. y Araldsen, H. (1997). Los niños que trabajan. Buenos Aires, UNICEF, Vol. 14. pp. 942.

Fernández, J. y De Los Campos, H. (2004). Análisis de las políticas y programas sociales en Uruguay: la acción pública para prevenir y combatir el trabajo de niños, niñas y adolescentes. Lima, OIT, Oficina Regional para las Américas / CIESU/IPEC Sudamérica. Documento de Trabajo 186, 176 pp.

Flórez, C.; Knaul, F. y Méndez, R. (1994). Un análisis cuantitativo del trabajo infantil y juvenil en Colombia. En: Revista Desarrollo y Sociedad. N.3 34, pp. 179-224.

Harsch, E. (2001). El trabajo infantil. Raíces en la pobreza de África. En: África Recovery. Vol. $15 \mathrm{~N}^{\circ} 3,14 \mathrm{pp}$.

Hernández, A. (2009). El trabajo infantil: una cuestión de derechos humanos. En: Revista De Estudios de la Violencia ICEV. ․․ 7, 23 pp.

Kassouf, A.; Mckee, M y Mossialos, E. (2001). Early Entrance to The Job Market and its Effect on Adult Health: Evidence from Brazil. En: Health Policy and Planning; Vol. 16. N. 1 , pp. 21-28.

Kolk, A. y Tulder, R. (2002). Child Labor and Multinational Conduct: A Comparison of International Business and Stakeholder Codes. En: Journal of Business Ethics. Vol. $36 \mathrm{~N}$. 3, pp. 291-301.

Mendoza, E. (2004). Trabajo infantil en la Central de Abastos de Bogotá, Corabastos. Diagnóstico general de la situación. Bogotá, OIT / IPEC. Sudamérica, Proyecto Prevención 
Erradicación del Trabajo Infantil en la Central de Abastos - Corabastos de Bogotá, Vol. 4 N. ${ }^{\circ} 2,88$ pp.

Moreno, C. (2003). Análisis cualitativo de la situación del trabajo infantil en Honduras. Tegucigalpa, Programa IPEC de la OIT para Centroamérica, 44 pp.

Mosquera, C.; Romero, M. Kathia y Huayhua, E. (1998). Proyecto erradicación progresiva del trabajo infantil en el caserío minero artesanal Santa Filomena. El riesgo de ser niño causas y efectos del trabajo infantil en el caserío minero artesanal Santa Filomena. Organización Internacional del Trabajo, Programa Internacional para la Erradicación del Trabajo Infantil, 19 pp.

OIT -Organización Internacional del Trabajo- (1973). Convenio No. 138 sobre la edad mínima de admisión al empleo. Ginebra, Programa Internacional para la Erradicación del Trabajo Infantil, Oficina Regional para América Latina y el Caribe, 7 pp.

OIT -Organización Internacional del Trabajo- (1999). Convenio 182 referente a las peores formas del trabajo infantil. Ginebra, Programa Internacional para la Erradicación del Trabajo Infantil, 4 pp.

OIT -Organización Internacional del Trabajo- (2001a). El trabajo infantil en la minería artesanal del oro. Diagnóstico sociocultural y económico del municipio de Condoto en Chocó. Bogotá, Programa Internacional para la Erradicación del Trabajo Infantil, OIT, Empresa Nacional Minera, 68 pp.

OIT -Organización Internacional del Trabajo- (2001b). El trabajo infantil en la minería artesanal del carbón del municipio de Sogamoso Boyacá. Bogotá, Programa Internacional para la Erradicación del Trabajo Infantil, Empresa Nacional Minera (Minercol), 63 pp.

OIT -Organización Internacional del Trabajo- (2001c). El trabajo infantil en la minería artesanal de esmeraldas. Diagnóstico sociocultural y económico del municipio de Muzo en Boyacá. Bogotá, Minercol; Sistema de Información Regional sobre Trabajo Infantil. — SIRTI — Oficina Regional para América Latina y el Caribe y Programa Internacional para la Erradicación del Trabajo Infantil, Sudamérica, 40 pp.

OIT -Organización Internacional del Trabajo- (2006). La Eliminación del Trabajo Infantil: Un Objetivo a Nuestro Alcance. Informe Global con Arreglo al Seguimiento de la Declaración de la OIT Relativa a los Principios y Derechos Fundamentales en el Trabajo. Conferencia Internacional del Trabajo 95ª Reunión, Informe I (B), 112 pp.

OIT -Organización Internacional del Trabajo- (2007a). Invertir en la familia. Estudio sobre factores preventivos y de vulnerabilidad de familias rurales y urbanas con hijos vinculados al trabajo doméstico en hogares de terceros: el caso Colombiano. Bogotá, Programa Internacional para la Erradicación del Trabajo Infantil, 165 pp.

OIT -Organización Internacional del Trabajo- (2007b). Trabajo infantil: causa y efecto de la perpetuación de la pobreza. San José, Programa Internacional para la Erradicación del Trabajo Infantil de la Oficina Internacional del Trabajo, 60 pp. 
OIT -Organización Internacional del Trabajo-. (2008). Introducción al Problema del Trabajo Infantil, Programa Internacional para la Erradicación del Trabajo Infantil, Oficina de Actividades para los Empleadores, 38 pp.

OIT -Organización Internacional del Trabajo-. (2009). Trabajo infantil y pueblos indígenas en América Latina: Una aproximación conceptual. Lima, Programa Internacional para la Erradicación del Trabajo Infantil, Oficina Regional para América Latina y el Caribe, 95 pp.

OIT -Organización Internacional del Trabajo- (2010). Entendiendo el Trabajo Infantil (UCW). Unidos en la lucha contra el trabajo infantil. La Haya, Informe Interagencial para la Conferencia Mundial Sobre Trabajo Infantil, 98 pp.

OIT -Organización Internacional del Trabajo- (2011). Niños en trabajos peligrosos: lo que sabemos, lo que debemos hacer. Ginebra, Programa Internacional para la Erradicación del Trabajo Infantil, Oficina Internacional del Trabajo, 116 pp.

ONU -Organización de las Naciones Unidas- (1989). Asamblea General. Resolución 44/25 del 20 de noviembre de 1989, 3 pp.

Pedraza, A. y Ribero, R. (2006). El trabajo infantil y juvenil en Colombia y algunas de sus consecuencias claves. En: Revista Latinoamericana de Ciencias Sociales, Niñez y Juventud, Vol. 4. N..$^{\circ}$, p p. 177-216.

Rodríguez, E. (2000). Capacitación y empleo de jóvenes en América Latina. Boletín Cinterfor OIT. Vol. 150, pp. 9-42.

Ruiz, E. (2001). Trabajo doméstico infantil y juvenil en los hogares ajenos: de la formulación de los derechos a su aplicación. Cuatro estudios locales en Colombia. Bogotá, UNICEF y Save The Children U. K. Colombia, 41 pp.

Straub R. y Rosati, F. (2006). Does Work during Childhood affect Adult's Health? An Analysis for Guatemala. Understanding Children's Work (UCW) Project University of Rome Tor Vergata, 18 pp.

UNICEF (2003). Trabajo infantil y adolecente en uruguay y su impacto sobre la educación. Análisis de la situación en la década pasada y el presente. Montevideo, Oficina de UNICEF en Uruguay, 43 pp.

Urueña, S.; Cuevas, L. y Castillo, M. (2009). Determinantes del trabajo infantil y la escolaridad: el caso del Valle del Cauca en Colombia. En: Revista Latinoamericana de Ciencias Sociales, Niñez y Juventud, Vol. 7 N.․ 2, pp. 707-733.

Vargas, S. (2005). Trabajo infantil urbano peligroso en Panamá: Un estudio de línea de base. San José, Organización Internacional del Trabajo, Programa Internacional para la Erradicación del Trabajo Infantil, Oficina Internacional del Trabajo, 205 pp.

Venegas, M. (2004). Erradicación del trabajo infantil en la industria pirotécnica de El Salvador. San José, International Programme on the Elimination of Child Labor, IPEC. Evaluación Final, 121 pp. 